\title{
Design and Application of Grey Predictor for Unglazed Transpired Solar Collector
}

\author{
Saliha Erenturk, Koksal Erenturk
}

\begin{abstract}
In order to evaluate different modeling techniques for Unglazed Transpired Collectors (UTC), not only mathematical modeling method for UTC based on heat transfer expressions to estimate the various heat transfer coefficients for the UTC components and empirical relationship, but also grey predicting approach have been designed and introduced, in this study. Thermal performance experiments of UTC have been carried out on an optimized experimental setup. Firstly, obtained experimental results have been compared with the mathematical model. To constitute a common point, output temperature of the UTC has been selected as the output variable. Then, a grey predictor has been used to forecast the output temperature with higher accuracy with the aid of simple mathematical equations. Finally, obtained results have been compared and comparison results have been illustrated in both graphical and tabular form. Grey predictor is the simplest method to forecast the output temperature with high accuracy.
\end{abstract}

Index Terms - Solar heating, grey modeling, grey predictor, unglazed transpired collectors.

\section{INTRODUCTION}

More sustainable and energy efficient building design and construction plays an important role in engineering of curtain wall assemblies [1]. In terms of thermal energy losses or gains, building envelope including different passive insulation methods is one of the most suitable method to mitigate thermal compensation requirements. For this purpose, passive solar walls called as UTC have been received increasing attention. UTC based air heating systems (AHS) have been installed on building envelope and manufactured as profit-oriented products in most of the developed countries [2]. UTC based passive solar AHS is one of the recent technological research concept for the cold climates, especially. Solar chimney [3], solar room [4], and trombe wall $[5,6]$ are some of the UTC based passive solar AHS. Hot air can be provided by using UTC system at the temperature range of $45-60 \mathrm{oC}$ for almost 200 days of the year with the 2664 hours/year sunshine period in Eastern region of Turkey [7].

UTC based passive solar air heating systems usually can be used for heating in winter. Despite the fact that the thermal load of envelope cannot be reduced with the usage of passive solar walls by controlling heat flux in summer, passive solar air heating technology can be taken into consideration as a special heating systems for Eastern region of Turkey because of the climate properties of the region.

Manuscript received October 09, 2018

Koksal Erenturk, Department of Computer Engineering, Ataturk University/ College of Engineering, Erzurum, Turkey, +90-542-255 1068, (erenturk@yahoo.com).

Saliha Erenturk, Department of Chemical Engineering, Ataturk University/ College of Engineering, Erzurum, Turkey, +90-442-231 4561, (serenturk@yahoo.com).
In order to determine the required amount of heat generation while the UTC based air heating systems are working, being able to predict the future values will be useful for reducing the energy consumption. With reducing energy consumption in the building using UTC heating system, most of the required conditions for more sustainable, more economic, eco-friendly building systems design will be satisfied. For this reason, if one can estimate or predict future values of UTC systems based on its behavior modeling, this approach will carry the studies on UTC systems to a step forward. As aforementioned reasons, to reduce the energy consumption grey modeling, ANN, and ANFIS based modeling techniques have been used to predict the future values of UTC system for different atmospheric conditions, in this study.

In systems theory point of view, "black box" expression generally used to define if any system has an incomplete information. Conversely, if all of the required information are accessible and complete this type system is called as "white". The meaning of "grey" can be used to express mixed characteristic neither black nor white. Generally, in real world applications, it is very hard to define the dynamic behavior of a complex system with a proper mathematical expression. Taking into consideration of a finite amount of available information, a new mathematical modeling technique based on grey modeling (GM) approach can be constituted to approximate the dynamic behavior of a system [8]. Therefore, in the literature, a great amount of published papers can be found for the applications of grey system in control and estimation [9-12].

This paper introduces efficient estimation algorithms to predict the output temperature of UTC air heating system based on grey modeling. As will be described later, not only quick response time and no cumulative prediction error but also no dependency on UTC parameters/characteristics and simplicity can be given attractive features of this estimation algorithm. The running performance evaluation of the proposed algorithm has been evaluated under different atmospheric and climatic conditions, with the inclusion of variations in the cloud factor, wind speed, relative humidity, and ambient temperature. Based on observed results, grey modeling approach has been found as the simplest method to predict the output temperature of UTC.

\section{EXPERIMENTAL SETUP AND MATHEMATICAL MODEL OF} UTC

Figure 1 depicts the prototype UTC air heating system currently under development. The designed collector consists of an absorber with holes and a back plate (plenum). The plenum and absorber has been separated with an air gap, and formed as a closed-box. A suction blower has been inserted at 
the top point of the box and has been used for the necessary suction during experimental runs. All of the experimental runs have been conducted on July 2016.

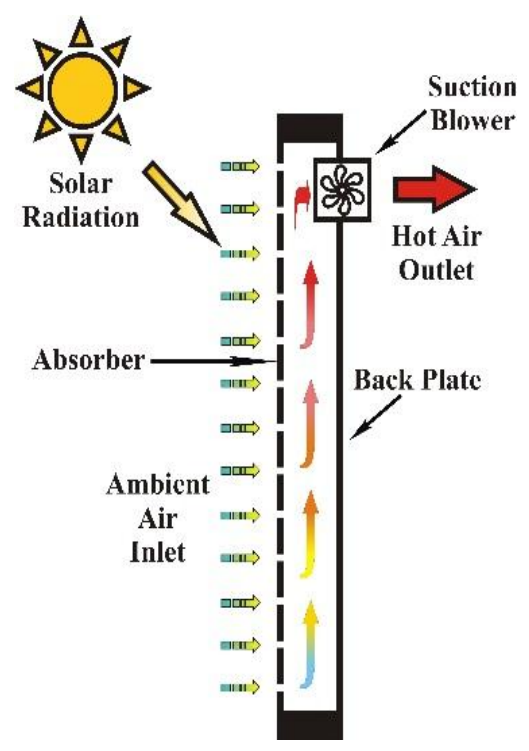

Figure 1. Schematic diagram of the UTC air heating system.

As illustrated in Fig. 2, the experiments have been conducted on an experimental collector. The collector has been manufactured from an aluminum absorber and its surface has been coated with black paint. The solar radiation range is within $400-750 \mathrm{~W} / \mathrm{m}^{2}$ for the experiments, which quite compatible with the mean of solar radiation in Eastern region in Turkey. The measured airflow rates are in the range of $0.5-2.5 \mathrm{~m} / \mathrm{s}$. The UTC experiments have been performed for different atmospheric conditions. The thickness of the collector is $1.0 \mathrm{~mm}$ and the dimensions of experimental collector are $77 \times 180 \mathrm{~cm}$. The depth of air gap is $10 \mathrm{~cm}$, perforation diameter is $1.5 \mathrm{~mm}$, and circular perforations in triangular pitch is assumed with $20 \mathrm{~mm}$.

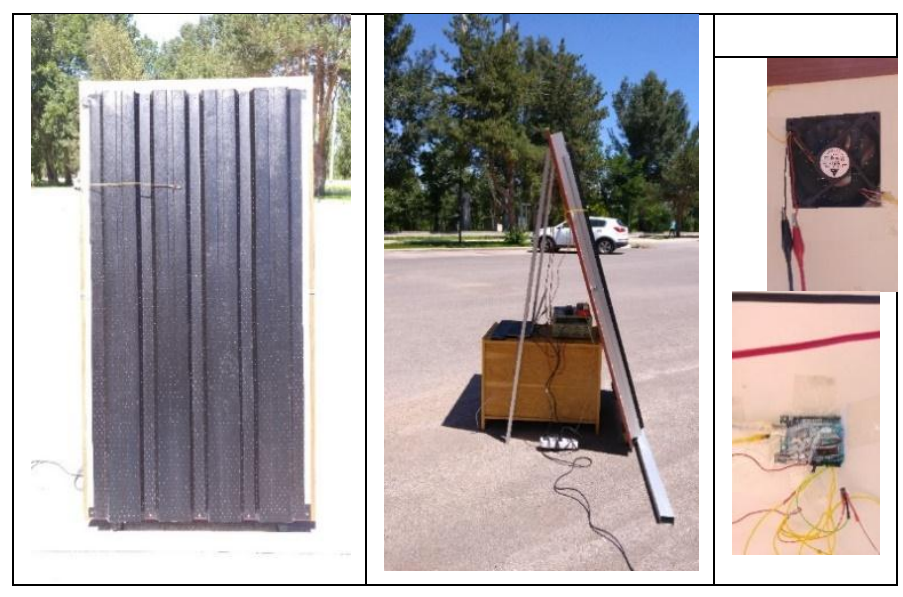

Figure 2. Experimental setup of the UTC air heating system.

Temperature measurements have been realized at 4 different points, such as: ambient temperature, output temperature, plenum temperature, and surface temperature, using LM35 temperature sensors. Measured temperatures have been stored in a laptop computer (Intel ${ }^{\circledR}$ Core $^{\mathrm{TM}}$ i7-2760QM CPU @ 2.40GHz, 8 GB RAM, 64-bit ACPI x64-based DELL PC) with the aid of an ARDUINO UNO data acquisition unit. The sampling rate of the ARDUINO has been selected as $1 \mathrm{~min}$. All of the data processing procedures, such as: grey modeling, ANN, and ANFIS estimation approaches, have been done in MATLAB® 2016b environment.

In order to constitute a model for the UTC mathematically, heat transfer in the UTC has been scrutinized by taken into consideration the whole energy equilibrium between the components of the collector. To calculate the heat transfer rates with convection and radiation between the components, rate equations have been used. A variety of heat transfer coefficients have been utilized in the rate equations to relate several empirical relations to the model. Absorber plate, air and the back plate are the essential components of the collector. The energy balance equations are derived from the solar heat input, thermal losses, and the heat and mass flow changes through the collector have been considered for the energy analysis.

The solar radiation on the UTC surface received are the main energy input to the UTC system. Thermal losses evolving on the UTC system are on account of convective and radiative type losses between the absorber surface and back plate.

$$
C_{a p} * m_{a p} * \frac{d T_{a p}}{d t}=(I * \alpha * A)-\left(Q_{r b p}+Q_{c a}+Q_{r s}\right)(1)
$$

where $C_{a p}$ is specific heat capacity of absorber material, $m_{a p}$ is mass of absorber plate, $T_{a p}$ is the temperature of absorber plate, $I$ is solar radiation incident on the collector, $\alpha$ is solar absorptance of the collector surface, $A$ is the collector area, $Q_{r b p}$ is the radiative heat transfer from the absorber to the back plate, $Q_{c a}$ is the heat gain from the absorber plate to air which includes heat transfer from the absorber front surface, hole and the back surface to the plenum air, $Q_{r s}$ is the radiative heat loss from the absorber surface to the surrounding, respectively.

Modeling of heat transfer for plenum air can be expressed as follow:

$$
\left(m_{\text {air }} * d t\right) * C_{a i r} * \frac{d T_{\text {air }}}{d t}=Q_{c a}-Q_{c b p}
$$

where $m_{\text {air }}$ is mass of air, $C_{\text {air }}$ is specific heat capacity of air, $T_{\text {air }}$ is the temperature of air, $Q_{c b p}$ is the heat flux from the air moving through the collector to the back plate, respectively.

If one want to estimate the radiation loss between the back plate and the surrounding, the surrounding temperature $T_{\text {sur }}$ can be calculated by means of the ground surface temperature and sky temperature.

$$
m_{b p} * C_{b p} * \frac{d T_{b p}}{d t}=\left(Q_{c b p}+Q_{r b p}\right)-\left(Q_{b p a m b}+Q_{r b p s}\right)
$$

where $m_{b p}$ is mass of the back plate, $C_{b p}$ is specific heat capacity of the back plate, $T_{b p}$ is the temperature of back plate, $Q_{\text {bpamb }}$ is the convection heat transfer between back plate and surrounding air, $Q_{r b p s}$ is radiation heat transfer between back plate and surrounding, respectively.

The rate equations can be divided into two-subcategories. One of them is "radiation heat transfer" while the other one is "convection heat transfer". As well-known, the heat transfer rates by the convection have been formulated using the convective heat transfer coefficient, heat transfer surface area, 
and the temperature difference between the surface and the circumambient fluid. Based on the previous approaches and to be able to use a single value for the plenum velocity in the calculations, an average value of the plenum velocity has been utilized instead of variable plenum velocity from the bottom to the top.

\section{GREY MODELING TECHNIQUE}

In terms of control applications, to be capable of calculating of the error values for the future sampling instants has a vitally importance for getting more powerful control operation when compared with the previously proposed classical or other control techniques. Grey modeling technique is one of the convenient methods for this purpose.

Using a data generation scheme to transform the irregular data into new data sets with strong regularity called as accumulated generating operation (AGO) are the essential operation principle of the grey system. It is obvious that, the more evidently data set can be defined by an exponential function for the more the times of accumulation.

In order to determine the grey model as in the form of GM $(n, h)$, the order of the differential equation, $n$, and the number of variables, $h$, should be defined. Commonly, $\operatorname{GM}(1,1)$ is the mostly used model-type for control applications in a real control environment and denotes a single-variable sequence and first-order linear grey model.

In order to use grey modeling operations in a real time applications, following information should be known to build $\operatorname{GM}(1,1)$ structure as described in Ref. [13].

The original data series with $n$ samples could be assumed as

$$
\Psi^{(0)}=\left\{\phi^{(0)}(1), \phi^{(0)}(2), \ldots \phi^{(0)}(n)\right\}
$$

The original data series of $\Psi$ has been represented by superscription (0). If the $1^{\text {st }}$-order accumulated generating operation applied to the $\Psi^{(0)}$, the following sequence could be expressed as

$$
\begin{aligned}
& \Psi^{(1)}=\left\{\phi^{(1)}(1), \phi^{(1)}(2), \ldots \phi^{(1)}(n)\right\} \\
& \text { in which } \phi^{(1)}(k) \text { is equal to } \\
& \phi^{(1)}(k)=\sum_{i=1}^{k} \phi^{(0)}(i), \quad k=1,2, \ldots, n
\end{aligned}
$$

In order to convert $\Psi^{(0)}$ into original values, inverse accumulated generating operation (IAGO) should be used. The conversion principle of the IAGO for the $\operatorname{GM}(1,1)$ type series can be defined as follows:

$$
\begin{aligned}
& \phi^{(0)}(1)=\phi^{(1)}(1), \text { and } \\
& \phi^{(0)}(k)=\phi^{(1)}(k)-\phi^{(1)}(k-1), \text { for } \mathrm{k}=2,3, \ldots, \mathrm{n}
\end{aligned}
$$

Based on the aforementioned basis for grey modeling technique, basic definition of the first-order grey differential equation can be expressed as below:

$$
\frac{d \Psi^{(1)}}{d t}+\alpha \Psi^{(1)}=\beta
$$

where $\alpha$ is a coefficient for the development and $\beta$ is the grey action quantity. For a grey system, the solution of the above differential equation will be resulted as prediction function. Then, the sequence $\hat{\phi}^{(1)}(k)$ will be:

$$
\hat{\phi}^{(1)}(k+1)=\left(\phi^{(0)}(1)-\frac{\beta}{\alpha}\right) e^{-\alpha k}+\frac{\beta}{\alpha},{ }_{k=1,2, \ldots, n(9)}
$$

The predicted value of $\phi$ is denoted by $\hat{\phi}$. Optimal and the best values for $\alpha$ and $\beta$ can be found by

$$
\left[\begin{array}{l}
\alpha \\
\beta
\end{array}\right]=\left(B^{T} B\right)^{-1} B^{T} Y
$$

where

$$
\begin{aligned}
Y & =\left[\phi^{(0)}(2), \phi^{(0)}(3), \ldots, \phi^{(0)}(n)\right]^{T} \\
B & =\left[\begin{array}{cc}
-\frac{1}{2}\left(\phi^{(1)}(2)+\phi^{(1)}(1)\right) & 1 \\
-\frac{1}{2}\left(\phi^{(1)}(3)+\phi^{(1)}(2)\right) & 1 \\
\vdots & \vdots \\
-\frac{1}{2}\left(\phi^{(1)}(n)+\phi^{(1)}(n-1)\right) & 1
\end{array}\right]
\end{aligned}
$$

The estimated value for the next step could be calculated as:

$$
\hat{\phi}^{(0)}(k+1)=\hat{\phi}^{(1)}(k+1)-\hat{\phi}^{(1)}(k)
$$

For all considered cases, $t$ has selected as bigger than 0 $(t>0)$. Accordingly, production of the negative sequence is impossible. In this study, $\operatorname{GM}(1,1)$ has been selected for the UTC modeling and to predict future values of the output temperature of the UTC.

\section{RESUlts}

Firstly, based on the whole energy equilibrium among the components of the UTC, a mathematical model for the UTC in terms of heat transfer has been scrutinized. As given in detail in mathematical model of UTC, in order to predict the convection and radiation type heat transfer rates among the components, related governing equations have been used. According to above mathematical modeling approach, following results that are given in Figure 3 have been obtained. That is why the existence of swings on the experimental data is based on the atmospheric and climatic conditions of the air. These swings have not been effected on the analysis since the developed algorithms are independent from the different atmospheric and climatic conditions, with the inclusion of variations in the cloud factor, wind speed, relative humidity, and ambient temperature. 


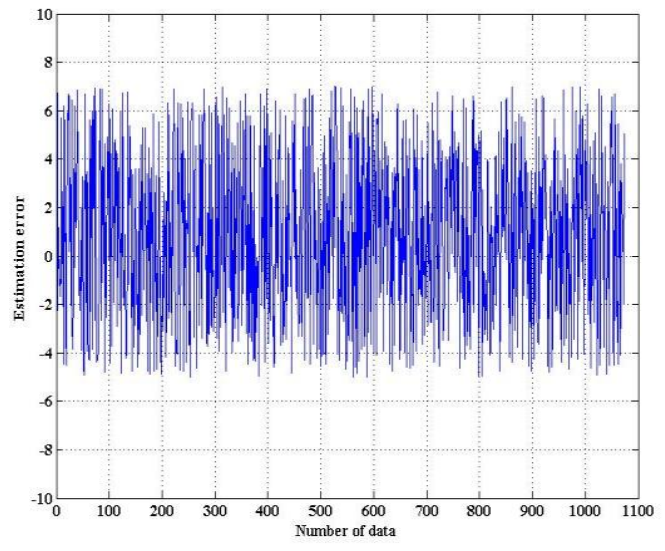

(a)

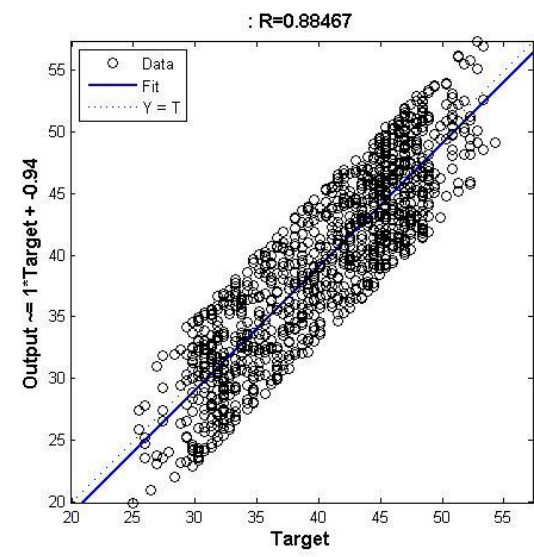

(b)

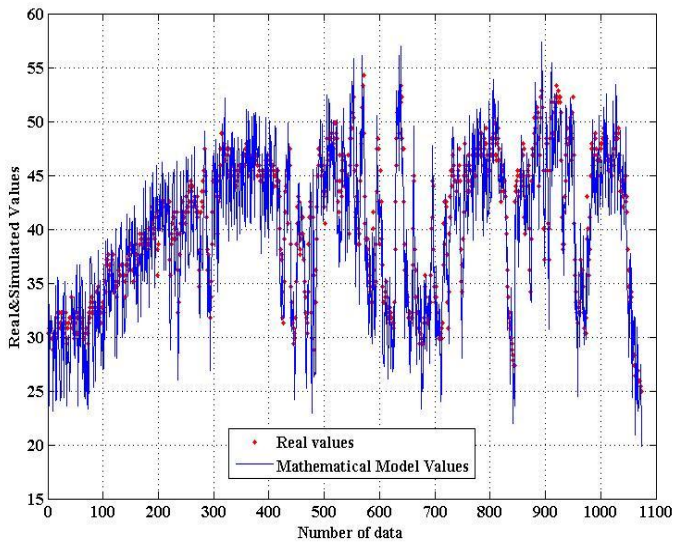

(c)

Figure 3. Results for mathematical modeling approach (a) Estimation error, (b) Regression analysis results, (c) Overlap illustration of the both real and predicted values.

As summarized in mathematical modeling of UTC section and as can be seen from Eq. 1-3, grey modeling technique is the one of the best approach to estimate future values of the output temperature of the UTC. Estimation error, regression analysis results for the estimation process, and comparison of the both real and predicted values have been represented in Figure 4.

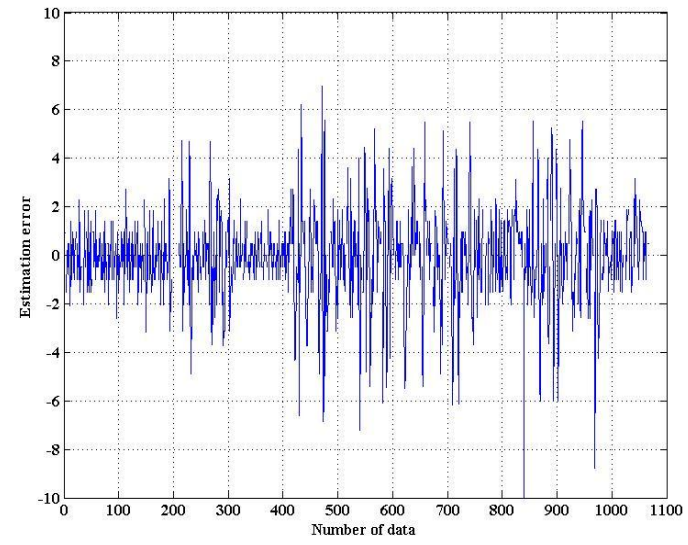

(a)

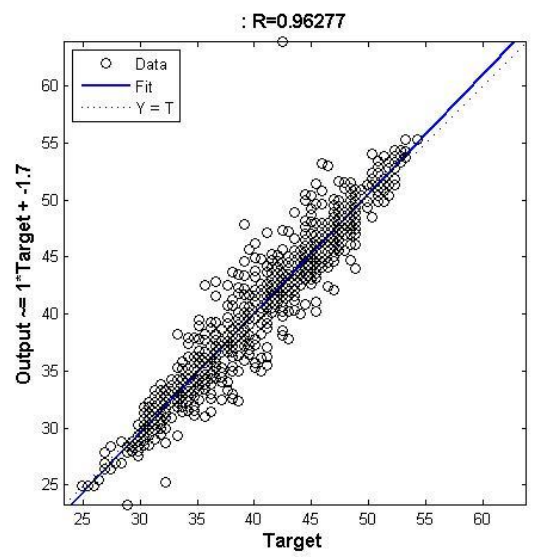

(b)

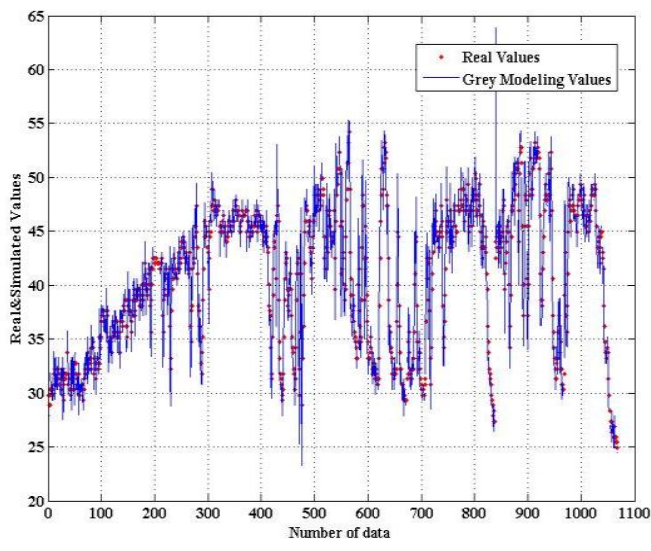

(c)

Figure 4. Grey modeling results (a) Estimation error, (b) Regression analysis results, (c) Overlap illustration of the both real and predicted values.

\section{CONCLUSION}

In order to estimate the output temperature of the UTC air heating system, brief introductions and design steps of grey modeling approach has been presented, in this study. An optimized experimental setup has been designed and thermal 
performance experiments of UTC have been carried out on this setup. According to the obtained results, it can be concluded that grey modeling approach is the simplest method to forecast the output temperature with high accuracy,

\section{REFERENCES}

[1] Sozer H. Improving energy efficiency through the design of the building envelope. Building and Environment 2010; 45 (12): 258193.

[2] SOLARWALL, 2016. The SOLARWALL Solar Heating System. Conserval Engineering Inc., Canada. Available from: $<$ http://www.solarwall.com/>. Accessed in August, 2016.

[3] Rakesh K, Lei C. An experimental investigation of an inclined passive wall solar chimney for natural ventilation. Solar Energy 2014; 107: 461-74.

[4] Feng J, Xin W, Yinping Z. A new method to estimate optimal phase change material characteristics in a passive solar room. Energy Conversion\&Management 2011; 52(6): 2437-41.

[5] Wei S, Jie J, Chenglong L, Wei H. Performance of PV-trombe wall in winter correlated with south facade design. Applied Energy 2011; 88(1): 224-31.

[6] Yanfeng L, Dengjia W, Chao M, Jiaping L. A numerical and experimental analysis of the air vent management and heat storage characteristics of a trombe wall. Solar Energy 2013; 91: 1-10.

[7] KUDAKA Technical Report, 2016. Available from: <http://www.kudaka.org.tr/e-dergi/Erzurum_Gunes_Enerjisi_Potansi yeli/>. Accessed in August, 2016.

[8] Deng JL. Introduction to grey system theory. Journal of Grey Systems 1989; 1(1): 1-24.

[9] Li Y, Zhao Y. The application of grey system theory to long-term prediction of weather. Journal of Grey Systems 1990; 2(1): 41-49.

[10] Tan C. Grey relational analysis of strong earthquake precursors. Journal of Grey Systems 1990; 2(1): 71-79.

[11] Deng JL. Control problems of grey systems. Systems and Control Letters 1982; 5: 228-294.

[12] Erenturk K. Hybrid Control of a Mechatronic System: Fuzzy Logic and Grey System Modeling Approach. IEEE/ASME Transactions on Mechatronics 2007; 12(6): 703-710.

[13] Liu SF, Lin Y. An Introduction to Gray Systems. Slippery Rock, PA: IIGSS Academic, 1998.

Koksal ERENTURK was born in Erzurum, Turkey, in 1973. He received his B.S. degree (with honors) from Yildiz Technical University, Istanbul, Turkey, in 1994. He also received his M.S. degree from Istanbul University, Istanbul, Turkey, in 1997, and Ph.D. degree from Karadeniz Technical University (Trabzon, Turkey), in 2002, all in electrical engineering. Currently, he is a Full Professor in the Department of Electrical and Electronics Engineering at Ataturk University, Erzurum, Turkey. His works have focused on the development and application of control theory to a variety of mechatronic systems, with a focus on observation and estimation based control. Much of his recent works have focused on the analysis and control of dynamical systems that arise in engineering applications. His areas of interest also include smart grids, fuzzy logic and fuzzy control, neural networks, genetic algorithms, and their applications to dynamic systems and electrical engineering.

Saliha ERENTURK is currently a Full Professor with the Department of Chemical Engineering, Ataturk University, Erzurum, Turkey. Her recent works have focused on the drying of various materials and their mathematical modeling, renewable energy and energy storage systems, heavy metal adsorption on the biomaterials, biofuels production and improving the some properties of biodiesel. 